\title{
Discovering Task Neighbourhoods through Landmark Learning Performances
}

\author{
Hilan Bensusan and Christophe Giraud-Carrier \\ Department of Computer Science, University of Bristol \\ Bristol BS8 1UB, United Kingdom \\ $\{$ hilanb, cgc\}@cs.bris.ac.uk
}

\begin{abstract}
Arguably, model selection is one of the major obstacles, and a key once solved, to the widespread use of machine learning/data mining technology in business. Landmarking is a novel and promising metalearning approach to model selection. It uses accuracy estimates from simple and efficient learners to describe tasks and subsequently construct meta-classifiers that predict which one of a set of more elaborate learning algorithms is appropriate for a given problem. Experiments show that landmarking compares favourably with the traditional statistical approach to meta-learning.
\end{abstract}

\section{Introduction}

The menu of data mining/machine learning techniques offers an increasingly large variety of choices. And, as with food, no one selection is universally satisfactory [15]. Instead, for every learning task, an appropriate learning algorithm must be chosen. An important goal of machine learning is to find principled ways to make this choice, which has become known as the model selection problem. Meta-learning endeavours to use machine learning itself (in a kind of recursive fashion) to the problem of model selection.

Meta-learning can be done by considering similar tasks in a reference class of tasks $[1,6,10,11]$, by inducing rules about algorithm selection $[7,11]$ or simply by training a meta-classifier $[2,3,9]$. In any case, tasks need to be described so that relations between tasks and learning algorithms can be automatically found. A number of strategies for task description have been used, based on statistics, information-theoretic considerations and properties of induced decision trees $[2$, $3,7,10,11,16]$. Recently, we have proposed a novel approach, called landmarking $[12,4]$. We believe this approach to be simpler, more intuitive and more effective.

Consider the class of tasks on which a learning algorithm performs well under a reasonable measure of performance (e.g., predictive accuracy) and call this class the area of expertise of the learner. The basic idea of landmarking is that the performance of a learner on a task uncovers information about the nature of that task. Hence, a task can be described by the collection of areas of expertise to which it belongs. We call a landmark learner, or simply a landmarker, a learning mechanism whose performance is used to describe a task. Exploring 
the meta-learning potential of landmarking amounts to investigating how well a landmark learner's performance hints at the location of learning tasks in the expertise space (i.e., the space of all areas of expertise). By thus locating tasks, landmarking discovers experimentally neighbouring areas of expertise. It therefore finds neighbourhoods of learning strategies and, ultimately, draws a map of the complete expertise space.

The paper is organised as follows. In section 2 we present our chosen set of landmarkers. Section 3 reports empirical results which complement those reported in $[12,4]$. Finally, section 4 concludes the paper.

\section{A Set of Landmarkers}

In principle, every learner's performance can signpost the location of a problem with respect to other learner's expertise. In practice, however, we want landmarkers that have low time complexity. ${ }^{1}$

Clearly, the landmarkers may change according to the learner pool, i.e., the set of target learners from which one must be selected. At present, our learner pools are subsets of $\{$ C5.0tree, C5.0rule, C5.0boost, MLC++'s Naive Bayes, MLC++'s IBL, Clementine's MLP, Clementine's RBF, Ripper, Linear Discriminant, Ltree\}. Given such pools, we construct the following set of landmarkers. In addition to being computationally efficient, each landmarker is intended to inform about the learning task, e.g., linear separability (see [4] for details).

1. Decision node: A single decision node consisting of the root of a C5.0 decision tree, where the attribute to branch on is chosen so as to maximise the information gain ratio [13].

2. Worst node: Same as above except that the attribute to branch on is chosen so as to minimise, rather than maximise, the information gain ratio.

3. Randomly chosen node: Same as above except that the attribute to branch on is chosen at random.

4. Naive Bayes: The standard Naive Bayes classifier [8].

5. 1-Nearest Neighbour: The standard 1-NN classifier, based on the closest training example [8].

6. Elite 1-Nearest Neighbour: Same as above except that the nearest neighbour is computed based on a subset of all attributes. This elite subset is composed of the most informative attributes if the information gain ratio difference between them is small. Otherwise, the elite subset is a singleton and the learner acts like a decision node learner.

7. Linear Discriminant: A linear approximation of the target function [14].

For each task, the error rate of a landmarker is given by the average error over the whole instance space of the learner trained on 10 different training sets of

\footnotetext{
${ }^{1}$ In general, the cost of running the chosen landmarkers should be smaller than the cost of running the more sophisticated learners; otherwise, it would be better to run all the learners to find out which one performs best.
} 


\begin{tabular}{|c||c|c|c|}
\hline Meta-learner & Landmarking & Information-based & Combined \\
\hline \hline Default Class & 0.460 & 0.460 & 0.460 \\
\hline C5.0boost & 0.248 & 0.360 & 0.295 \\
\hline C5.0rules & 0.239 & 0.333 & 0.301 \\
\hline C5.0tree & 0.242 & 0.342 & 0.314 \\
\hline MLP & 0.301 & 0.317 & 0.320 \\
\hline RBFN & 0.289 & 0.323 & 0.304 \\
\hline LD & 0.335 & 0.311 & 0.301 \\
\hline Ltree & 0.270 & 0.317 & 0.286 \\
\hline IB & 0.329 & 0.366 & 0.342 \\
\hline NB & 0.429 & 0.407 & 0.363 \\
\hline Ripper & 0.292 & 0.314 & 0.295 \\
\hline \hline Average & 0.298 & 0.339 & 0.312 \\
\hline
\end{tabular}

Table 1. Meta-learning performance for different task descriptions

equal size. Each task is then described by a vector of 7 meta-attributes (i.e., the error rates of the 7 landmarkers) and labelled by the learner (from the learner pool), which gives the highest predictive accuracy based on stratified 10 -fold cross-validation. We use the special label "tie" when the difference in performance between the best and worst learners is small (here, less than $10 \%)^{2}{ }^{2}$ Once a (meta-)dataset has thus been constructed, it can be (meta-)learned by any supervised learning technique.

\section{Experiments with Landmarking}

In the first experiment, we compare landmarking with the more traditional statistical/information theoretic approach to task description (e.g., see [9, 11]). We consider the following 6 widely-used meta-attributes: class entropy, average entropy of the attributes, mutual information, joint entropy, equivalent number of attributes and signal-to-noise ratio. For this comparative experiment, the learner pool includes all 10 learners. We use 320 artificially generated Boolean datasets with 5 to 12 attributes, where the classifications are partly rule-based and partly random. The 10 learning algorithms in the learner pool are also used as meta-learners. Error rates are based on stratified 10-fold cross-validation and the results are given in Table 1 . The default class is "tie".

Table 1 shows that landmarking compares favourably with the informationbased task description approach. In all but 2 cases, landmarking produces lower error rates than the information-based approach. The table also suggests that adding the information-based features to describe task impairs landmarking performance. These results are further confirmed by the learning curves of the three approaches (see Fig. 1).

\footnotetext{
${ }^{2}$ When performance differences are small, meta-learning is not only harder but also
} less useful since there is no great advantage of the best learner over the others. 


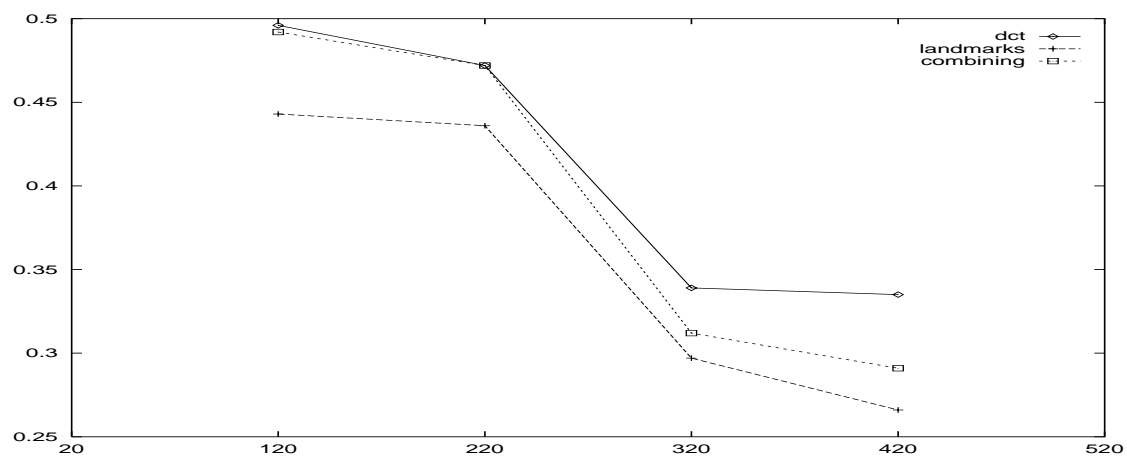

Fig. 1. Learning curves for the landmarking approach, the information-based approach and the combined approach

Note that in the above experiment three learners, namely Linear discriminant, Naive Bayes and Nearest Neighbor, are both in the set of landmarkers and in the learner pool. This can be seen as making the landmarking enterprise too easy. Notice, however, that the meta-learners are not informed of this identity, nor is it easy to uncover it since the landmarking meta-attributes values are only error rates. In fact, the meta-learner has to proceed as in the general case, finding correlations between the meta-attributes and the best learners. Experiments reported in [4], for example, suggest that the presence of Naive Bayes among the landmarkers does not significantly impact performance.

In the second experiment, we consider the performance of landmarking when the learner pool is smaller. Of course, if the learner pool is sufficiently small, it is more efficient to run all the learners in the pool to select the most suitable one. However, this experiment is intended to shed some light on the ability of landmarking to describe tasks and discriminate between areas of expertise. We restrict our attention to pairs of learners including C5.0boost (C5B), MLP, Ripper (RIP) and Naive Bayes (N-B). The results, reported in Table 2, show that landmarking with most meta-learners improves on the default class.

In the third experiment, we investigate whether knowledge induced from artificial tasks is valid when applied to more "real-world" applications. To this end, we train C4.5 (as a meta-learner) on the meta-dataset arising from our 320 Boolean problems and test the induced hypothesis on 16 benchmark datasets from UCI [5] and Daimler-Chrysler. Table 3 reports the error rate differences between the best and the worst learners. The figures preceded by $\nu$ are the error figures of cases where landmarking chooses the best learner. Hence, these are figures of errors that landmarking avoids. When landmarking selects "tie", we pessimistically make the assumption that it would select the worst learner. The results show that, in most cases, landmarking's recommendations are either right or not very damaging to the overall performance. This is to say that it selects the worst learner when the worst learner is not greatly worse than the best one. 


\begin{tabular}{|c||c|c|c|c|c|}
\hline Meta-learner & C5B vs MLP & C5B vs RIP & MLP vs RIP & N-B vs RIP & C5B vs N-B \\
\hline \hline Default Class & 0.450 & 0.550 & 0.510 & 0.490 & 0.550 \\
\hline C5.0boost & 0.328 & 0.308 & 0.366 & 0.176 & 0.193 \\
\hline C5.0rules & 0.355 & 0.317 & 0.411 & 0.138 & 0.179 \\
\hline C5.0tree & 0.364 & 0.334 & 0.405 & 0.147 & 0.176 \\
\hline MLP & 0.387 & 0.358 & 0.472 & 0.252 & 0.235 \\
\hline RBFN & 0.410 & 0.331 & 0.481 & 0.276 & 0.261 \\
\hline LD & 0.375 & 0.364 & 0.425 & 0.267 & 0.290 \\
\hline Ltree & 0.384 & 0.249 & 0.405 & 0.199 & 0.188 \\
\hline IB & 0.387 & 0.308 & 0.405 & 0.278 & 0.264 \\
\hline NB & 0.554 & 0.387 & 0.627 & 0.375 & 0.334 \\
\hline Ripper & 0.364 & 0.317 & 0.446 & 0.202 & 0.199 \\
\hline \hline Average & 0.391 & 0.327 & 0.444 & 0.231 & 0.232 \\
\hline
\end{tabular}

Table 2. Landmarking performance when selecting between pairs of learners

\section{Conclusions}

Landmarking is a strategy to describe tasks through the performance of simple and efficient learners. Tasks are therefore described by their position in the expertise space. The expertise space is itself a suggestive way of thinking of learners and tasks. Landmarking relies on the position of a problem in that space to represent it: "tell me what can learn you and I will tell you what you are". Empirical results suggest that it pays off to run bare-bone, landmark learners on a number of tasks and learn how their performance relates to that of other, more fleshed-out learners. These results indicate that the information about a task given by the performance of a portfolio of simple learners is useful for meta-learning. Furthermore, they also suggest that it is safe to apply to real-world tasks, knowledge learned from artificial ones.

\section{Acknowledgements}

This work is supported by an ESPRIT Framework IV LTR Grant (Nr. 26.357). We thank the anonymous referees for useful comments.

\section{References}

1. D.W. Aha. Generalizing from case studies: A case study. In Proceedings of 9th ICML, pages 1-10, 1992.

2. H. Bensusan. God doesn't always shave with Occam's Razor - learning when and how to prune. In Proceedings of 10th ECML, pages 119-124, 1998.

3. H. Bensusan. Automatic bias learning: an inquiry into the inductive basis of induction. PhD thesis, School of Cognitive and Computing Sciences, University of Sussex, 1999. 


\begin{tabular}{|c||c|c|r|r|r|}
\hline Task & C5B vs MLP & C5B vs RIP & MLP vs RIP & N-B vs RIP & C5B vs N-B \\
\hline \hline mushrooms & $\nu 0.050$ & 0.000 & $\nu 0.032$ & $\nu 0.003$ & 0.003 \\
\hline abalone & $\nu 0.500$ & 0.033 & 0.088 & 0.047 & 0.015 \\
\hline crx & 0.056 & $\nu 0.027$ & 0.008 & 0.084 & $\nu 0.110$ \\
\hline acetylation & $\nu 0.091$ & $\nu 0.357$ & $\nu 0.267$ & $\nu 0.333$ & $\nu 0.024$ \\
\hline titanic & 0.007 & 0.211 & 0.219 & $\nu 0.216$ & 0.004 \\
\hline waveform & 0.040 & 0.176 & $\nu 0.136$ & $\nu 0.204$ & 0.028 \\
\hline yeast & 0.009 & 0.276 & $\nu 0.428$ & $\nu 0.422$ & 0.004 \\
\hline car & 0.011 & $\nu 0.083$ & 0.094 & $\nu 0.021$ & $\nu 0.104$ \\
\hline chess & $\nu 0.112$ & $\nu 0.380$ & 0.269 & 0.195 & $\nu 0.186$ \\
\hline led7 & $\nu 0.014$ & $\nu 0.042$ & 0.028 & 0.038 & 0.004 \\
\hline led24 & 0.013 & $\nu 0.001$ & 0.012 & 0.025 & 0.026 \\
\hline tic & 0.009 & $\nu 0.005$ & $\nu 0.015$ & $\nu 0.280$ & $\nu 0.274$ \\
\hline MONK1 & $\nu 0.156$ & $\nu 0.256$ & 0.000 & 0.218 & 0.245 \\
\hline MONK2 & $\nu 0.141$ & $\nu 0.141$ & 0.000 & $\nu 0.164$ & $\nu 0.023$ \\
\hline satimage & $\nu 0.431$ & $\nu 0.046$ & 0.016 & $\nu 0.068$ & $\nu 0.114$ \\
\hline quisclas & 0.023 & $\nu 0.003$ & 0.026 & $\nu 0.258$ & $\nu 0.261$ \\
\hline
\end{tabular}

Table 3. Error rate differences between best and worst learners

4. H. Bensusan and C. Giraud-Carrier. Casa batló is in passeig de gràcia or landmarking the expertise space. In Proceedings of ECML-2000 Workshop on MetaLearning: Building Automatic Advice Strategies for Model Selection and Method Combination, pages 29-46, 2000.

5. C.L. Blake and C.J. Merz. UCI repository of machine learning databases, 1998 http://www.ics.uci.edu/ mlearn/MLRepository.html.

6. P. Brazdil and C. Soares. A comparison of ranking methods for classification algorithm selection. In Proceedings of 11th ECML, 2000.

7. C. E. Brodley. Recursive automatic bias selection for classifier construction. $M a-$ chine Learning, 20:63-94, 1995.

8. R. O. Duda and P. E. Hart. Pattern classification and scene analysis. Wiley, 1973.

9. R. Engels and C. Theusinger. Using a data metric for offering preprocessing advice in data mining applications. In Proceedings of 13th ECAI, pages 430-434, 1998.

10. G. Lindner and R. Studer. AST: Support for algorithm selection with a CBR approach. In Proceedings of 3rd PKDD, pages 418-423, 1999. (LNAI 1704).

11. D. Michie, D. J. Spiegelhalter, and C. C. Taylor, editors. Machine Learning, Neural and Statistical Classification. Ellis Horwood, 1994.

12. B. Pfahringer, H. Bensusan, and C. Giraud-Carrier. Meta-learning by landmarking various learning algorithms. In Proceedings of 17th $I C M L$, pages 743-750, 2000

13. J. R. Quinlan. C4.5: Programs for Machine Learning. Morgan Kaufmann, 1993.

14. B. D. Ripley. Pattern Recognition and Neural Networks. Cambridge University Press, 1995.

15. C. Schaffer. A conservation law for generalization performance. In Proceedings of 11th ICML, pages 259-265, 1994.

16. G. Widmer. On-line metalearning in changing context: MetaL(b) and metaL(ib). In Proceedings of 3rd International Workshop on Multistrategy Learning, 1997. 